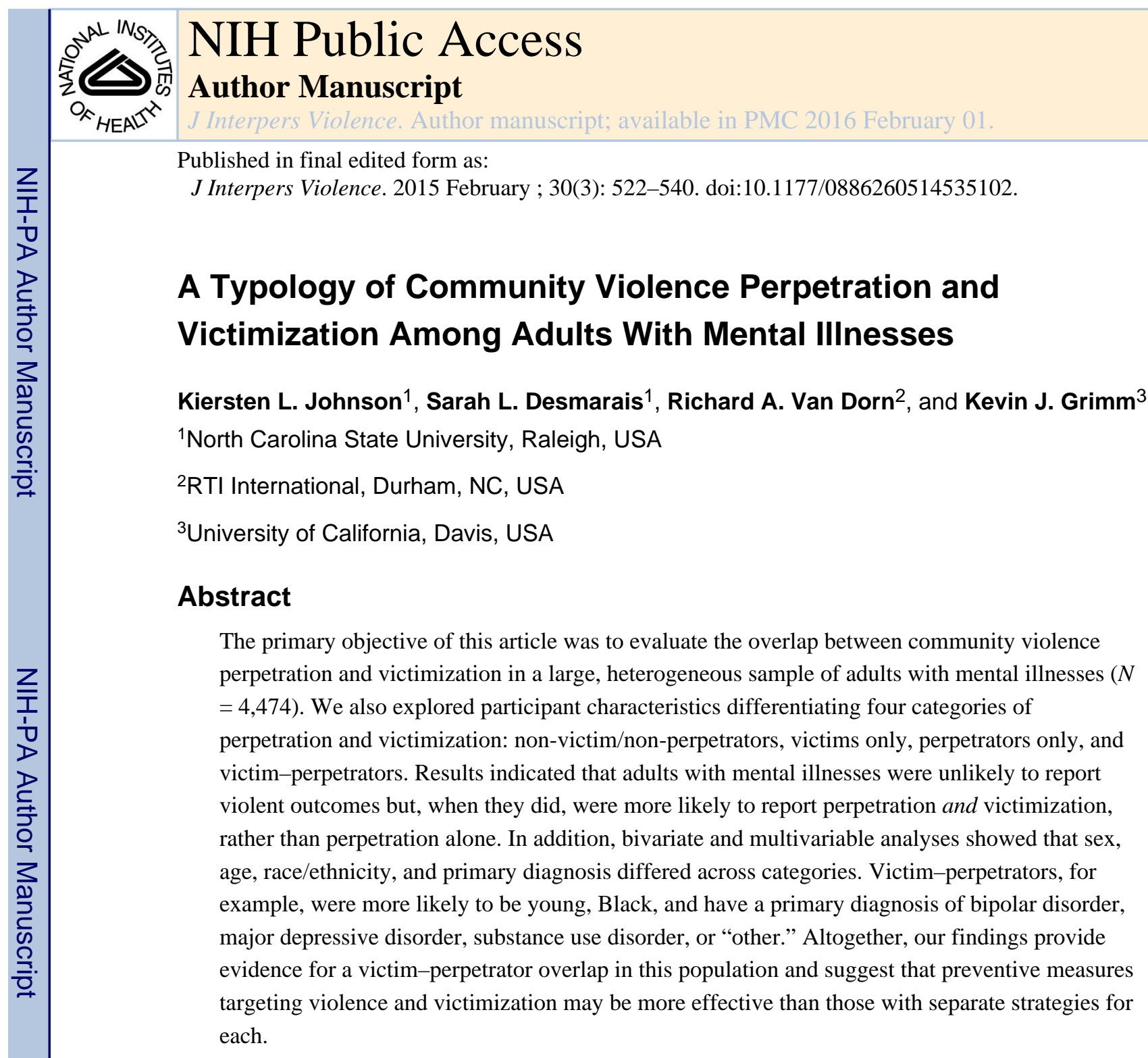

\title{
Keywords
}

mental health and violence; community violence; violence exposure

\begin{abstract}
Many view adults with mental illnesses as potentially violent and a threat to public safety (Corrigan \& Watson, 2002). Although evidence indicates that there is a modest increased risk of violence associated with mental illness compared with the general public (Corrigan \& Watson, 2005; Swanson, Holzer, Ganju, \& Jono, 1990; Van Dorn, Volavka, \& Johnson, 2012), adults with mental illnesses also are more likely to be victimized than the general public (Goodman, Thompson, \& Weinfurt, 1999; Teplin, McClelland, Abram, \& Weiner, 2005). However, research examining community violence perpe-tration and victimization in adults with mental illnesses has typically focused on only one of these outcomes. In the
\end{abstract}

(C) The Author(s) 2014

Corresponding Author: Kiersten L. Johnson, Department of Psychology, North Carolina State University, Campus Box 7650, Raleigh, NC 27695-7650, USA. kljeske@ncsu.edu.

Declaration of Conflicting Interests

The author(s) declared no potential conflicts of interest with respect to the research, authorship, and/or publication of this article. 
present study, we examine the overlap between community violence perpetration and victimization, as well as identify participant characteristics that differentiate individuals across four categories: non-victim/non-perpetrators, victims only, perpetrators only, and victim-perpetrators.

\section{Community Violence Perpetration and Victimization in Adults With Mental Illnesses}

Only a handful of papers have examined community violence perpetration and victimization within the same sample during the same time frame. One study, for example, assessed 419 adults entering short-term residential treatment (Havassy \& Mericle, 2013). Close to half of the sample (40.8\%) reported violence in the past 30 days, with $84.2 \%$ of these incidents including victimization. Another study assessed 331 adults with mental illnesses (53.8\% male) who were under outpatient commitment orders. Prevalence of violence was 50.4\% and of victimization (violent and non-violent), 27.2\%. Multivariable regression analyses indicated that each incident of victimization was associated with a 1.76 increase in the odds of violence (Hiday, Swanson, Swartz, Borum, \& Wagner, 2001). A 3-year study of 172 adults (73.8\% male) with schizophrenia measuring incidence of perpetration and victimization found that $34.0 \%$ of the sample reported being victimized (violent and nonviolent), while only $6.4 \%$ were charged for violence against others. Associations between perpetration and victimization were not reported (Brekke, Prindle, Bae, \& Long, 2001). A fourth study examined community violence perpetration and victimization 10 weeks after discharge in a sample of 826 discharged psychiatric patients (58\% male), finding that $19.4 \%$ of respondents reported victimization only, $13.0 \%$ reported perpetration only, and $5.6 \%$ reported both perpetration and victimization. There was a significant association between violence and victimization $(r=.46, p<.05$; Silver, Piquero, Jennings, Piquero, \& Lieber, 2011).

Together, findings of prior studies show lower rates of perpetration than victimization among adults with mental illnesses and consistently show that perpetration and victimization occur among the same individuals within the same time frames. Although results are informative, they also are limited in several ways. Generalizability of some studies is restricted by small, non-representative samples, as well as heterogeneity in operational definitions and measurement of perpetration and victimization across, but also within, certain studies. Moreover, prior studies have not always focused on the association between perpetration and victimization, nor have they examined correlates within a given period of time.

Even though violence perpetration and victimization are not often measured within the same sample and/or time frame, extant research demonstrates that prior violence or victimization increases risk of the other. For example, victimization has been identified as one of the most robust correlates of perpetration among adults with mental illnesses (Swanson et al., 2002; Witt, Van Dorn, \& Fazel, 2013). Likewise, prior violent offending has been shown to increase risk for violent victimization in this population (Silver, 2002). This trend is mirrored in the general population (Wittebrood \& Nieuwbeerta, 1999). All told, findings from prior research suggest that preventive measures intended to decrease risk for one 
violent outcome may serve to reduce risk for the other in adults with mental illnesses. Thus, the overlap between violence perpetration and victimization in this vulnerable population merits further investigation.

\section{Victim-Perpetrator Overlap in the General Population}

In contrast with the scant research conducted with adults with mental illnesses, there have been many studies on the association between perpetration and victimization in the general population. A recent review of 37 studies found that the majority $(83.8 \%, n=31)$ showed a significant statistical association between perpetration and victimization (Jennings, Piquero, \& Reingle, 2012). Specifically, the two were positively and significantly related across a variety of settings, age groups, and types of outcomes (e.g., non-violent, violent). Indeed, the association was stronger for violent offenses (e.g., homicide) compared with non-violent offenses (e.g., property crime), but was nonetheless present for the latter.

Although most prior studies dichotomized violent offending and victimization, a handful grouped participants as a function of outcomes. For example, one study of 9,421 adolescents and young adults categorized participants as non-violent (71.3\%), perpetrators only (4.7\%), victims only (12.0\%), and victim-perpetrators (11.9\%; Reingle, Staras, Jennings, Branchini, $\&$ Maldonado-Molina, 2011). Another multi-wave study of 1,138 Puerto Rican youth found that non-victim, non-offenders represented from $31.3 \%$ to $36.6 \%$ of the sample, victims from $32.4 \%$ to $43.8 \%$, offenders from $4.3 \%$ to $9.1 \%$, and victim-offenders from $15.3 \%$ to $27.4 \%$ (Maldonado-Molina, Jennings, Tobler, Piquero, \& Canino, 2010). Findings of these studies further suggest that participant characteristics may distinguish between groups. To demonstrate, male and Black youth in the Reingle et al. sample were significantly more likely than their counterparts to be victims of intimate partner violence. Similarly, repeat victim-offenders in the Maldonado-Molina et al. sample were more likely to be male and older compared with the rest of the sample.

\section{The Present Study}

Few studies have examined the overlap between community violence perpetration and victimization in adults with mental illnesses, despite evidence that these two outcomes have shared associations (Daday, Broidy, Crandall, \& Sklar, 2005; Wittebrood \& Nieuwbeerta, 1999) and act as risk factors for each other (Swanson et al., 2002; Witt et al., 2013). To that end, our primary research objective was to evaluate the overlap between community violence perpetration and victimization in adults with mental illnesses. Our secondary objective sought to identify participant characteristics (i.e., sex, age, race/ethnicity, primary diagnosis) that differentiated categories of violence perpetration and victimization.

\section{Method}

\section{Data}

Baseline data were pooled from five studies of adults with mental illnesses: (a) Facilitated Psychiatric Advance Directive (F-PAD) Study ( $n=469$; Swanson et al., 2006); (b) MacArthur Mental Disorder and Violence Risk (MacRisk) Study ( $n=1,136$; Steadman et 
al., 1998); (c) Schizophrenia Care and Assessment Program (SCAP) Study ( $n=404$; Swanson, Swartz, \& Elbogen, 2004); (d) MacArthur Mandated Community Treatment (MacMandate) Study ( $n=1,011$; Monahan et al., 2005); and (e) Clinical Antipsychotic Trials of Intervention Effectiveness (CATIE) Study ( $n=1,460$; Lieberman et al., 2005). A total of 4,474 adults with mental illnesses are included in the present analyses. We briefly describe each data source below.

F-PAD study - This randomized clinical trial examined the implementation of a facilitated PAD program. A random sample of clients prescreened for eligibility was drawn from a deidentified list from two mental health systems in North Carolina. Upon providing informed consent, participants were randomly assigned to either the intervention or control group and administered a semi-structured interview regarding PADs prior to and following the intervention. Inclusion criteria were (a) 18 to 65 years of age; (b) schizophrenia-spectrum or major mood disorder; and (c) currently in treatment.

MacRisk study-This study assessed violence risk of civil psychiatric patients. Eligible participants were sampled from three acute inpatient facilities, with recruitment adjusted to maintain a consistent distribution of age, sex, and ethnicity across sites. Inclusion criteria were (a) English-speaking White, Black, or Hispanic patients; (b) 18 to 40 years of age; and (c) schizophrenia-spectrum, depression, mania, brief reactive psychosis, or delusional disorder. Data were collected through participant and collateral interviews and hospital abstractions from 1992 to 1995.

SCAP study-This study assessed clinical, functional, and service utilization outcomes for adults with schizophrenia. Participants were recruited from treatment facilities across North Carolina using both sequential inpatient admissions and a random selection of outpatients, with eligibility limited to (a) 18 years of age or older; (b) schizophrenia; and (c) current service use. Data were collected through in-person interviews at 6-month intervals and medical record abstraction over a 3-year period.

MacMandate study-This study collected data regarding lifetime experience of leverage (e.g., housing, criminal justice, and outpatient commitment) from outpatients through structured interviews and clinic charts between 2002 and 2003. Participants were recruited through both sequential and random selection across sites, with eligibility limited to (a) 18 to 65 years of age; (b) English- or Spanish-speaking; (c) current outpatient treatment; and (d) first service occurred at least 6 months prior.

CATIE study-This randomized clinical trial examined the effectiveness of second generation antipsychotic medication in adults with schizophrenia. Participants were recruited from 57 sites (16 university clinics, 10 state mental health agencies, 7 Veteran's Affairs Medical Centers, 6 private nonprofit agencies, 4 private practice sites, and 14 mixed system sites) across the United States. Inclusion criteria were (a) 18 to 65 years of age; (b) schizophrenia; and (c) ability to take oral antipsychotics. Data were collected through selfreport, individual/collateral interviews, and clinician ratings in monthly visits over an 18month period. 


\section{Measures}

Dependent variables-Prevalence of community violence perpetration and victimization was assessed in all studies using the MacArthur Community Violence Screening Instrument (MCVSI; Steadman et al., 1998). The MCVSI is comprised of eight questions each for violence and victimization, derived from the Revised Conflict Tactics Scale (CTS2; Straus, Hamby, Boney-McCoy, \& Sugarman, 1996). The questions assess (a) pushing, grabbing, or shoving; (b) kicking, biting, or choking; (c) slapping; (d) throwing an object; (e) hitting with a fist or object; (f) sexual assault; (g) threatening with a weapon in hand; and (h) using a weapon. Total administration time is approximately 5 to $7 \mathrm{~min}$. For the present analyses, we dichotomized responses of "violence" and "other aggressive acts" to obtain prevalence of violent outcomes (yes/no) in the past 6 months.

Independent variables-Participant sex is measured dichotomously with 1 representing female and 0 representing male. Age is a measured continuously (in years) and reflects age at baseline. Racelethnicity is captured with four indicator variables: White, Black, Hispanic, and other/mixed race/ethnicity, with 1 representing group membership and 0 representing non-membership. Primary diagnosis is measured with five indicator variables: schizophrenia, bipolar disorder, major depressive disorder, substance use disorder, and other diagnosis, with 1 representing primary diagnosis and 0 representing absence or secondary diagnosis. Diagnoses were obtained through a combination of clinician diagnoses and medical records, with recent diagnoses by a psychiatrist in a medical record taking precedence over any prior diagnoses. We controlled for differences across studies by modeling study effects with a series of indicator variables.

\section{Data Analysis}

All analyses were conducted with SPSS, v.19. Descriptive statistics were calculated for all variables. To address our primary research objective, we identified participants' group membership based on their responses to the MCVSI (e.g., those who responded "yes" to violent outcomes in both violence and victimization scales were classified as victimperpetrators). Across categories, we computed frequencies and percentages for categorical variables (sex, race, diagnosis), and means and standard deviations for age. Our secondary objective was addressed through bivariate and multivariable analyses. Specifically, to examine bivariate associations between categorical variables and groups, we conducted chisquare tests; post hoc pairwise comparisons were conducted using Bonferroni adjusted $z$ tests. For age, we conducted a one-way ANOVA with group membership as the independent variable; post hoc pairwise comparisons were conducted using Bonferroni adjusted $t$ tests. We additionally ran pairwise comparisons to examine if prevalence of experiencing both violence and victimization in cases where at least one violent outcome was reported differed as a function of participant characteristics. Multivariable analyses were conducted using multinomial logistic regression. Odds ratios show probability of membership in each group, as compared with the non-victim, non-perpetrator group. For primary diagnosis and race/ ethnicity, schizophrenic and White participants, respectively, served as reference groups. For the study covariate, the CATIE study served as the reference group. 


\section{Results}

\section{Descriptive Statistics}

Table 1 presents participant characteristics overall and across the violence and victimization categories. In the sample, the majority of participants were male and White. Participant age ranged between 18 and 71 years. Schizophrenia was the most common primary diagnosis, followed by major depression, bipolar disorder, substance use disorder, and other (e.g., anxiety) disorders.

Table 1 also shows the prevalence of violent outcomes. Participants were more likely to report no victimization or violence than any other category. Victim-perpetrators comprised the next largest group, but represented less than one fifth of the sample, followed by victims only and perpetrators only. Distribution of categories differed across studies, reflecting the variation in patients sampled across study sites.

\section{Bivariate Analyses}

Bivariate analyses showed that the distribution of male and female participants differed significantly across categories. Post hoc $z$ tests indicated that the perpetrator-only group deviated significantly from the 2:1 male-to-female ratio present in the other categories, and, in fact, had a greater representation of women than men. Race/ethnicity also differed across categories. Specifically, Hispanic participants were less likely to identify as victimperpetrators compared with White and Black respondents. Age also differed significantly across categories. Post hoc comparisons showed that mean age for each category differed in all cases except between victims only and perpetrators only. On average non-victim/nonperpetrators were the oldest, while victim-perpetrators were the youngest.

Primary diagnoses differed across categories. Participants with schizophrenia were more likely to identify as non-victim/non-perpetrators and less likely to identify as victimperpetrators than those with any other diagnosis. They were less likely to identify as victims only than participants with bipolar or substance use disorder; furthermore, they were less likely to identify as perpetrators only than participants with major depressive or "other" disorders. Conversely, participants with substance use disorder were less likely to identify as non-victim/non-perpetrators and more likely to identify as victim-perpetrators than any other diagnosis.

Table 2 presents the prevalence of experiencing both violence and victimization when least one has been reported. Overall, results demonstrate that across all participants, nearly half of those reporting any violence or victimization experienced both in the 6-month time frame. Pairwise comparisons revealed some differences in the proportion of participants reporting both violence and victimization as a function of participant characteristics. Specifically, of participants reporting any instance of violence or victimization, Hispanic participants were less likely than White participants to experience both in the 6-month time frame. In addition, compared with participants with major depressive or "other" disorders, those with schizophrenia were significantly less likely to experience both. The percentage of men and women who reported both violence and victimization in the 6-month time frame did not differ significantly. 


\section{Multivariable Analyses}

Table 3 presents the results of our multinomial logistic regression. Overall, there was adequate discrimination among categories on the basis of the four correlates: $-2 \log$ Likelihood $=5,383.65, \chi^{2}(39, N=4455)=816.38, p<.001$, Nagelkerke $R^{2}=.19$. In the sections that follow, we describe factors associated with membership in each category.

Perpetrator-only—All four variables—age, sex, race/ethnicity, and primary diagnosissignificantly distinguished perpetrators only from non-victim/non-perpetrators. Younger participants were more likely to be in this group. Male participants were less likely than female participants to identify as perpetrators only than as non-victim/non-perpetrators. Black, Hispanic, and "other/mixed" race/ethnicity participants were nearly twice as likely as White participants to identify as perpetrators only than as non-victim/non-perpetrators. Similarly, compared with participants with a primary diagnosis of schizophrenia, participants with major depressive disorder or "other" disorders were more likely to identify as perpetrators only.

Victim-only-Only age and race/ethnicity distinguished between non-victim/nonperpetrator and victim-only groups. Specifically, younger age significantly increased the likelihood of being in the victim-only group. Compared with White participants, participants belonging to "other/mixed" racial/ethnic groups were more than twice as likely to identify as victims only than as non-victim/non-perpetrators.

Victim-perpetrator-Age, race/ethnicity, and primary diagnosis significantly distinguished between non-victim/non-perpetrators and victim-perpetrators. Younger age significantly increased the likelihood of identifying as a victim-perpetrator. Compared with White participants, Black participants were more likely to identify as victim-perpetrators than as non-victim/non-perpetrators. Compared with participants with a primary diagnosis of schizophrenia, those with bipolar disorder, major depressive disorder, substance use disorder, and "other" diagnosis were more likely to identify as victim-perpetrators. Specifically, participants with primary diagnoses of substance use disorder and "other" diagnosis were roughly 3 times more likely to identify as victim-perpetrators than those with schizophrenia.

\section{Discussion}

Adults with mental illnesses are at heightened risk for violence, both perpetration (Corrigan $\&$ Watson, 2005; Van Dorn et al., 2012) and victimization (Goodman et al., 1999; Teplin et al., 2005); however, few studies have had the capability and/or objective to identify an overlap between the two violent outcomes. Thus, the present study's primary objective was to examine the victim-perpetrator overlap in a large, pooled sample of adults with mental illnesses. We additionally identified characteristics associated with non-victim/nonperpetrators, victims only, perpetrators only, and victim-perpetrators. Below, we summarize these findings. 


\section{Summary of Findings}

Overall, and contrary to public opinion, our findings indicate that the majority of adults with mental illnesses were neither victims nor perpetrators of violence during the reference period. Specifically, almost two thirds of the sample (62.9\%) reported no violent incidents. In fact, only $6.3 \%$ of participants reported violence perpetration but not victimization. This rate is less than half the rate seen for victimization only (13.3\%). Notably, participants were more likely to report experiencing both violent perpetration and victimization in the 6-month period $(17.6 \%)$ than they were to report either perpetration or victimization alone. These rates are consistent with a prior analysis of the MacArthur Violence Risk Study data that examined both perpetration and victimization (Silver et al., 2011) and lend support to the existence of a victim-perpetrator overlap in this population. Furthermore, our findings indicate that adults with mental illnesses experience violence-both perpetration and victimization - at rates higher than reported in the general population, as has been previeusly suggested (Steadman et al., 1998, Teplin et al., 2005). As such, continued emphasis on violence without acknowledgment of the increased risk of victimization presents an incomplete picture of the data.

Findings of our bivariate and multivariable analyses help to address our secondary research objective and to add additional clarity to previous research findings. Overall, participant characteristics distinguished between groups in meaningful ways. For example, analyses demonstrated that younger age is consistently associated with violent outcomes-both perpetration and victimization, alone or together-as has been seen in prior research conducted with the general population (Maldonado-Molina et al., 2010; Reingle et al., 2011) and adults with mental illnesses (Swanson et al., 1990; Teplin et al., 2005). This study is one of few to show the association vis-à-vis victim-perpetrators, whether in adults with mental illnesses (Silver et al., 2011) or other populations (e.g., Maldonado-Molina et al., 2010; Reingle et al., 2011).

Our analyses also showed that White participants were more likely to be in the non-victim, non-perpetrator group than any other group. Conversely, non-White participants were more likely to be victims and/or perpetrators, though associations and group membership differed by specific racial/ethnic minority group. However, of participants reporting any instance of violence or victimization, Hispanic participants were less likely than White participants to experience both violence and victimization in the 6-month time frame. These results are consistent with some prior research in which Black (Silver et al., 2011) and Hispanic (Corrigan \& Watson, 2005) adults with mental illnesses are more likely than their White counterparts to be perpetrators of violence. Black adults with mental illnesses additionally are more likely to be victims of violence (Silver, 2002; Teplin et al., 2005). That said, results of other studies of adults with mental illnesses have been mixed in terms of the risk of violence perpetration and victimization associated with minority racial/ethnic status (e.g., Brekke et al., 2001; Hiday et al., 2001; Silver et al., 2011). Herein we have shown that not only are racial and ethnic minorities at increased risk for violent outcomes but also that they are at increased risk for both perpetration and victimization during the same period of time.

As with age and race/ethnicity, primary diagnosis was significantly related to group membership in our bivariate and multivariable analyses. Specifically, participants with a 
primary diagnosis of schizophrenia were significantly less likely than participants with other primary diagnoses to commit violence, either as perpetrators only or victim-perpetrators. Adults with major depressive disorder and "other" primary diagnoses, however, were at heightened risk of violence, both as perpetrators only and victim-perpetrators; they were also more likely than those with a primary diagnosis of schizophrenia to experience both outcomes if either was reported.

Although most studies find co-occurring substance use disorder increases risk for violence perpetration (Swanson et al., 1990; Swanson et al., 2006; Van Dorn et al., 2012) and victimization (Goodman et al., 2001) in adults with mental illnesses, a primary diagnosis of substance use disorder was not associated with perpetration only or victimization only. It did, however, increase likelihood of membership in the victim-perpetrator group. These results may reflect an overall increase in adverse outcomes in adults with a primary diagnosis of substance use disorder. Indeed, substance use is widely recognized as a risk factor for adverse outcomes among adults with mental illnesses (Swanson et al., 2006; Van Dorn et al., 2012).

Sex was significantly related to membership in just one group: perpetration only. Results of the multinomial logistic regression showed that women were more likely than men to be in the perpetrator-only group compared with the non-perpetrator, non-victim group. This finding is generally inconsistent with prior studies of both adults with mental illnesses (Van Dorn et al., 2012; Witt et al., 2013) and the general population (Monahan, 1997; Stueve \& Link, 1998). However, the sex differences we found may reflect our operationalization of violence. Specifically, research attributing higher overall rates of violence perpetration to women also reports similar or higher rates of more serious acts of violence among men (Desmarais et al., 2014; Monahan et al., 2001). Moreover, the higher rates of female violence may reflect our use of self-report rather than official records (e.g., police reports). That said, compared with the general population, the sex difference may be smaller or reversed for adults with mental illnesses (Hiday, Swartz, Swanson, Borum, \& Wagner, 1998; Lidz, Mulvey, \& Gardner, 1993; Short, Thomas, Mullen, \& Ogloff, 2013; Stueve \& Link, 1998). Findings also may reflect increased opportunity: Women with mental illnesses are more likely to live with family members and men to live alone (Swanson et al., 2006). Indeed, in another analysis of the present data (Desmarais et al., 2014), the majority of violent incidents were perpetrated in residential settings.

Our identification of the relative prevalence of and individual characteristics associated with categories of violence perpetration and victimization guide the interpretation of previously equivocal findings. That adults with mental illnesses are at greater risk for violence and victimization rather than either individual outcome alone provides evidence for a victimoffender overlap in this population and suggests a new direction in policy and practice. Specifically, interventions and community initiatives targeting violence reduction often have separate strategies for victims and offenders (Daday et al., 2005). However, practitioners and programs may achieve more success by targeting individuals with the shared characteristics of victim-perpetrators, such as substance use disorder. In addition, our finding that adults with a primary diagnosis of schizophrenia are less likely than those with other primary diagnoses to perpetrate violence contradicts the public and structural stigmas 
that have been found to lead clinicians to override patients' treatment wishes in cases of perceived violence risk (Swanson, Van McCrary, Swartz, Van Dorn, \& Elbogen, 2007), as well as prevent adults with mental illnesses from seeking or fully participating in mental health services (Corrigan, 2004). Overall, our findings contribute to mounting evidence against such stigmas and can thus aid in improving public perceptions of adults with schizophrenia and, furthermore, increase service use in this population. Finally, from an epidemiological point of view, our findings of the characteristics associated with the different categories of violence perpetration and victimization can assist in the identification of higher risk populations to be targeted for early intervention.

\section{Limitations and Future Directions}

The present study has several limitations. First, we analyzed cross-sectional data. General population research suggests that the overlap between violence and victimization may change over time (Maldonado-Molina et al., 2010); however, we did not examine causal or longitudinal effects. Thus, future research should implement a prospective design to assess longitudinal trends, as well as causality among variables. Second, we focused on demographic factors and primary diagnosis, but there are many other factors that may prove useful in differentiating between groups. Future research should incorporate additional factors, such as socioeconomic status, social support, and access to treatment. Third, with our focus on primary diagnoses, we did not include any indication of co-occurring substance use disorders, which are known to heighten risk for both violence perpetration and victimization (e.g., Swanson et al., 2006; Van Dorn et al., 2012). Further exploration of these factors, as well as co-occurring disorders, may add to our understanding the overlap between perpetration and victimization in adults with mental illnesses. Fourth, our data were derived from self-report and may be susceptible to the effects of social desirability, recall bias, and errors. The reconstruction of violent incidents in semi-structured interviews is inherently incomplete (Estroff, Swanson, Lachicotte, Swartz, \& Bolduc, 1998), and though self-report is a valid and reliable measure for collecting data on violence perpetration and victimization (Huizinga \& Elliot, 1986; Van Dorn et al., 2010), additional sources, such as hospital and arrest records, may capture non-self-reported violent events. Fifth, data on interrater reliability are not available, though descriptions of interviewer training are provided elsewhere (Monahan et al., 2001; Stroup et al., 2003).

\section{General Conclusion}

This article marks a first step in understanding the overlap between community violence perpetration and victimization in adults with mental illnesses. Findings show that the four categories of violence perpetration and victimization identified in the general population non-victim/non-perpetrators; perpetrators only; victims only; and victim-perpetrators - are also seen in adults with mental illnesses at similar rates. Findings additionally indicate that adults with mental illnesses are more likely not to report violent outcomes but, when they do, are more likely to report both violence perpetration and victimization rather than perpetration alone. The distinct characteristics associated with the different groups emphasize that adults with mental illnesses are a heterogeneous population, and that young, 
non-White adults with primary diagnoses other than schizophrenia are particularly vulnerable to violent outcomes.

\section{Acknowledgments}

Funding

The author(s) received no financial support for the research, authorship, and/or publication of this article.

\section{Author Biographies}

Kiersten L. Johnson, MS, is a third-year doctoral student in the Psychology in the Public Interest program at North Carolina State University. Her research interests include violence risk assessment, as well as correlates of substance use, violence, and victimization in adults with mental illness.

Sarah L. Desmarais, $\mathrm{PhD}$, is an assistant professor of Psychology in the Public Interest at North Carolina State University. Her program of work is focused on the development, implementation, and evaluation of assessment and intervention strategies for justiceinvolved adolescents and adults with behavioral health problems.

Richard A. Van Dorn, $\mathrm{PhD}$, is a senior mental health services researcher at RTI International. His program of research is focused on violence and victimization among adults with mental illness.

Kevin J. Grimm, $\mathrm{PhD}$, is an associate professor of psychology at the University of California, Davis. His program of research is focused on the development and application of statistical models for longitudinal data and data integration.

\section{References}

Brekke JS, Prindle C, Bae SW, Long JD. Risks for individuals with schizophrenia who are living in the community. Psychiatric Services. 2001; 52:1358-1366. [PubMed: 11585953]

Corrigan PW. How stigma interferes with mental health care. American Psychologist. 2004; 59:614625. [PubMed: 15491256]

Corrigan PW, Watson AC. Understanding the impact of stigma on people with mental illness. World Psychiatry. 2002; 1:16-20. [PubMed: 16946807]

Corrigan PW, Watson AC. Findings from the National Comorbidity Survey on the frequency of violent behavior in individuals with psychiatric disorders. Psychiatry Research. 2005; 136:153-162. [PubMed: 16125786]

Daday JK, Broidy LM, Crandall CS, Sklar DP. Individual, neighborhood, and situational factors associated with violent victimization and offending. Criminal Justice Studies. 2005; 18:215-235.

Desmarais SL, Van Dorn RA, Johnson KL, Grimm KJ, Douglas KS, Swartz MS. Community violence perpetration and victimization among adults with mental illness. American Journal of Public Health. Feb 13.2014 Advance online publication.

Estroff SE, Swanson JW, Lachicotte WS, Swartz M, Bolduc M. Risk reconsidered: Targets of violence in the social networks of people with serious psychiatric disorders. Social Psychiatry \& Psychiatric Epidemiology. 1998; 33:S95-S101. [PubMed: 9857786]

Goodman LA, Salyers MP, Mueser KT, Rosenberg SD, Swartz M, Essock SM, Swanson J. Recent victimization in women and men with severe mental illness: Prevalence and correlates. Journal of Traumatic Stress. 2001; 14:615-632. [PubMed: 11776413] 
Goodman LA, Thompson K, Weinfurt K. Reliability of violent victimization and PTSD among men and women with serious mental illness. Journal of Traumatic Stress. 1999; 12:587-599. [PubMed: 10646178]

Havassy BE, Mericle AA. Recent violence among persons entering short-term residential mental health and substance abuse treatment. Journal of Dual Diagnosis. 2013; 9:222-227.

Hiday VA, Swanson JW, Swartz MS, Borum R, Wagner HR. Victimization: A link between mental illness and violence? International Journal of Law and Psychiatry. 2001; 24:559-572. [PubMed: 11795220]

Hiday VA, Swartz MS, Swanson JW, Borum R, Wagner HR. Male-female differences in the setting and construction of violence among people with severe mental illness. Social Psychiatry \& Psychiatric Epidemiology. 1998; 33:S68-S74. [PubMed: 9857782]

Huizinga D, Elliott DS. Reassessing the reliability and validity of self-report delinquency measures. Journal of Quantitative Criminology. 1986; 2:293-327.

Jennings WG, Piquero AR, Reingle JM. On the overlap between victimization and offending: A review of the literature. Aggression and Violent Behavior. 2012; 17:16-26.

Lidz C, Mulvey E, Gardner W. The accuracy of predictions of violence to others. Journal of the American Medical Association. 1993; 269:1007-1011. [PubMed: 8429581]

Lieberman JA, Stroup TS, McEvoy JP, Swartz MS, Rosenheck RA, Perkins DO, Hsiao JK. Effectiveness of antipsychotic drugs in patients with chronic schizophrenia. New England Journal of Medicine. 2005; 353:1209-1223. [PubMed: 16172203]

Maldonado-Molina MM, Jennings WG, Tobler AL, Piquero AR, Canino G. Assessing victim-offender overlap among Hispanic youth. Journal of Criminal Justice. 2010; 38:1191-1201.

Monahan, J. Clinical and actuarial predictions of violence. In: Faigman, D.; Kaye, DH.; Saks, MJ.; Sanders, J., editors. Modern scientific evidence: The law and science of expert testimony. West; St. Paul, MN: 1997. p. 300-318.

Monahan J, Redlich A, Swanson JW, Robbins PC, Appelbaum PS, Petrila J, McNiel DE. Use of leverage to improve adherence to psychiatric treatment in the community. Psychiatric Services. 2005; 56:37-44. [PubMed: 15637190]

Monahan, J.; Steadman, HJ.; Silver, E.; Appelbaum, P.; Robbins, PC.; Mulvey, EP.; Banks, S. Rethinking risk assessment: The MacArthur Study of Mental Disorder and Violence Risk. Oxford University Press; New York, NY: 2001.

Reingle JM, Staras SAS, Jennings WG, Branchini J, Maldonado-Molina MM. The relationship between marijuana use and intimate partner violence in a nationally representative, longitudinal sample. Journal of Interpersonal Violence. 2011; 27:1562-1578. [PubMed: 22080574]

Short T, Thomas S, Mullen P, Ogloff JRP. Comparing violence in schizophrenia patients with and without comorbid substance-use disorders to community controls. Acta Psychiatrica Scandinavica. 2013; 128:306-313. [PubMed: 23379839]

Silver E. Mental disorder and violent victimization: The mediating role of involvement in conflicted social relationships. Criminology. 2002; 40:191-212.

Silver E, Piquero AR, Jennings WG, Piquero N, Lieber M. Assessing the violent offending and violent victimization overlap among discharged psychiatric patients. Law and Human Behavior. 2011; 35:49-59. [PubMed: 20145985]

Steadman HJ, Mulvey EP, Monahan J, Robbins PC, Appelbaum PS, Grisso T, Silver E. Violence by people discharged from acute psychiatric inpatient facilities and by others in the same neighborhoods. Archives of General Psychiatry. 1998; 55:393-401. [PubMed: 9596041]

Straus MA, Hamby SL, Boney-McCoy S, Sugarman DB. The Revised Conflict Tactics Scales (CTS2): Development and preliminary psychometric data. Journal of Family Issues. 1996; 17:283-316.

Stroup TS, McEvoy JP, Swartz MS, Byerly MJ, Glick ID, Canive JM, Lieberman JA. The National Institute of Mental Health Clinical Antipsychotic Trials of Intervention Effectiveness (CATIE) project: Schizophrenia trial design and protocol development. Schizophrenia Bulletin. 2003; 29:15-31. [PubMed: 12908658]

Stueve A, Link BG. Gender differences in the relationship between mental illness and violence: Evidence from a community-based epidemiological study in Israel. Social Psychiatry \& Psychiatric Epidemiology. 1998; 33:S61-S67. [PubMed: 9857781] 
Swanson JW, Holzer CE, Ganju VK, Jono RT. Violence and psychiatric disorders in the community: Evidence from the Epidemiologic Catchment Area Surveys. Hospital \& Community Psychiatry. 1990; 41:761-770. [PubMed: 2142118]

Swanson JW, Swartz MS, Elbogen EB. Effectiveness of atypical antipsychotic medications in reducing violent behavior among persons with schizophrenia in community-based treatment. Schizophrenia Bulletin. 2004; 30:3-20. [PubMed: 15176758]

Swanson JW, Swartz MS, Elbogen EB, Van Dorn RA, Ferron J, Wagner HR, Kim M. Facilitated psychiatric advance directives: A randomized trial of an intervention to foster advance treatment planning among persons with severe mental illness. American Journal of Psychiatry. 2006; 163:1943-1951. [PubMed: 17074946]

Swanson JW, Swartz MS, Essock SM, Osher FC, Wagner HR, Goodman LA, Meador KG. The socialenvironmental context of violent behavior in persons treated for severe mental illness. American Journal of Public Health. 2002; 92:1523-1531. [PubMed: 12197987]

Swanson JW, Van McCrary S, Swartz MS, Van Dorn RA, Elbogen EB. Overriding psychiatric advance directives: Factors associated with psychiatrists' decisions to preempt patients' advance refusal of hospitalization and medication. Law and Human Behavior. 2007; 31:77-90. [PubMed: $16718578]$

Teplin LA, McClelland GM, Abram KM, Weiner DA. Crime victimization in adults with severe mental illness: Comparison with the National Crime Victimization Survey. Archives of General Psychiatry. 2005; 62:911-921. [PubMed: 16061769]

Van Dorn R, Volavka J, Johnson N. Mental disorder and violence: Is there a relationship beyond substance use? Social Psychiatry \& Psychiatric Epidemiology. 2012; 47:487-503. [PubMed: 21359532]

Van Dorn RA, Kosterman R, Williams JH, Chandler K, Young MS, Catalano RF, Hawkins JD. The relationship between outpatient mental health treatment and subsequent mental health symptoms and disorders in young adults. Administration and Policy in Mental Health and Mental Health Services Research. 2010; 37:484-496. [PubMed: 20186567]

Witt K, Van Dorn RA, Fazel S. Risk factors for violence in psychosis: Systematic review and metaregression analyses of 110 studies. PLoS ONE. 2013; 8:1-15.

Wittebrood K, Nieuwbeerta P. Wages of sin? The link between offending, lifestyle, and violent victimization. European Journal on Criminal Policy and Research. 1999; 7:63-80. 


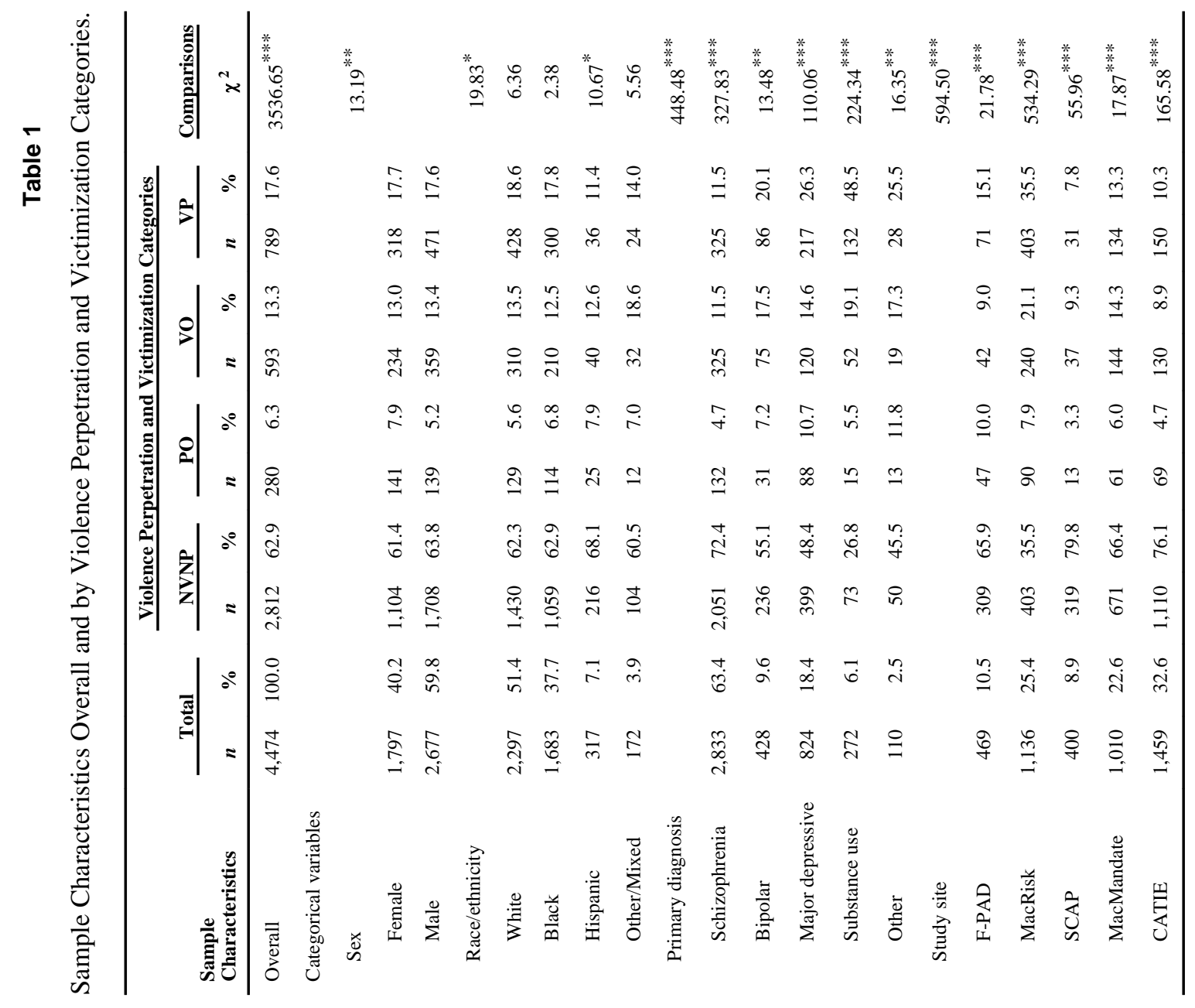




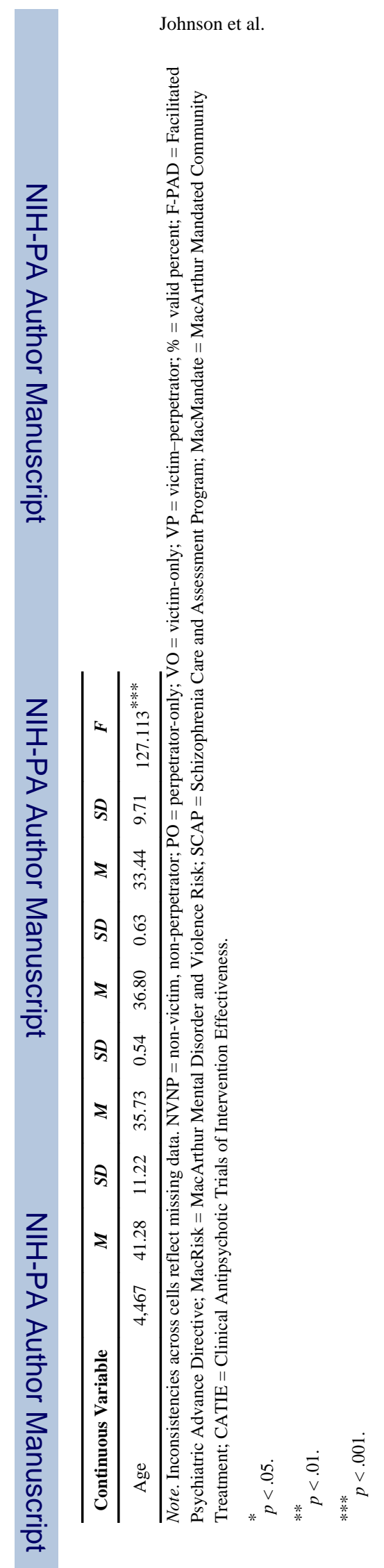

Page 15 
Table 2

Prevalence of Both Violence and Victimization When Any Violent Outcome Was Reported.

\begin{tabular}{lccc}
\hline & $\begin{array}{c}\text { \% Reporting } \\
\text { Any Violence or } \\
\text { Victimization }\end{array}$ & $\begin{array}{c}\text { \% Reporting } \\
\text { Both Violence } \\
\text { and Victimization }\end{array}$ & Comparisons \\
\hline Overall & 37.1 & 47.5 & \\
Sex & 38.6 & 45.9 & - \\
$\quad$ Female & 36.2 & 48.6 & 1.77 \\
$\quad$ Male & & & \\
Race/ethnicity & 37.7 & 49.4 & - \\
White (reference) & 37.1 & 48.1 & 0.81 \\
Black & 31.9 & 35.6 & $4.61^{* * *}$ \\
Hispanic & 39.5 & 52.9 & 0.89 \\
Other/Mixed & & & - \\
Primary diagnosis & 27.6 & 41.6 & 1.25 \\
Schizophrenia (reference) & 44.9 & 44.8 & $4.84^{* * *}$ \\
Bipolar & 51.6 & 51.1 & $7.94^{* * *}$ \\
Major depressive & 73.2 & 66.3 & 1.04 \\
Substance use & 54.5 & 46.7 & \\
Other & & & \\
\hline
\end{tabular}

Note. $\%$ = valid percent.

$\stackrel{*}{p}<.05$.

$* * \quad p<.01$.

$* * * x<.001$. 


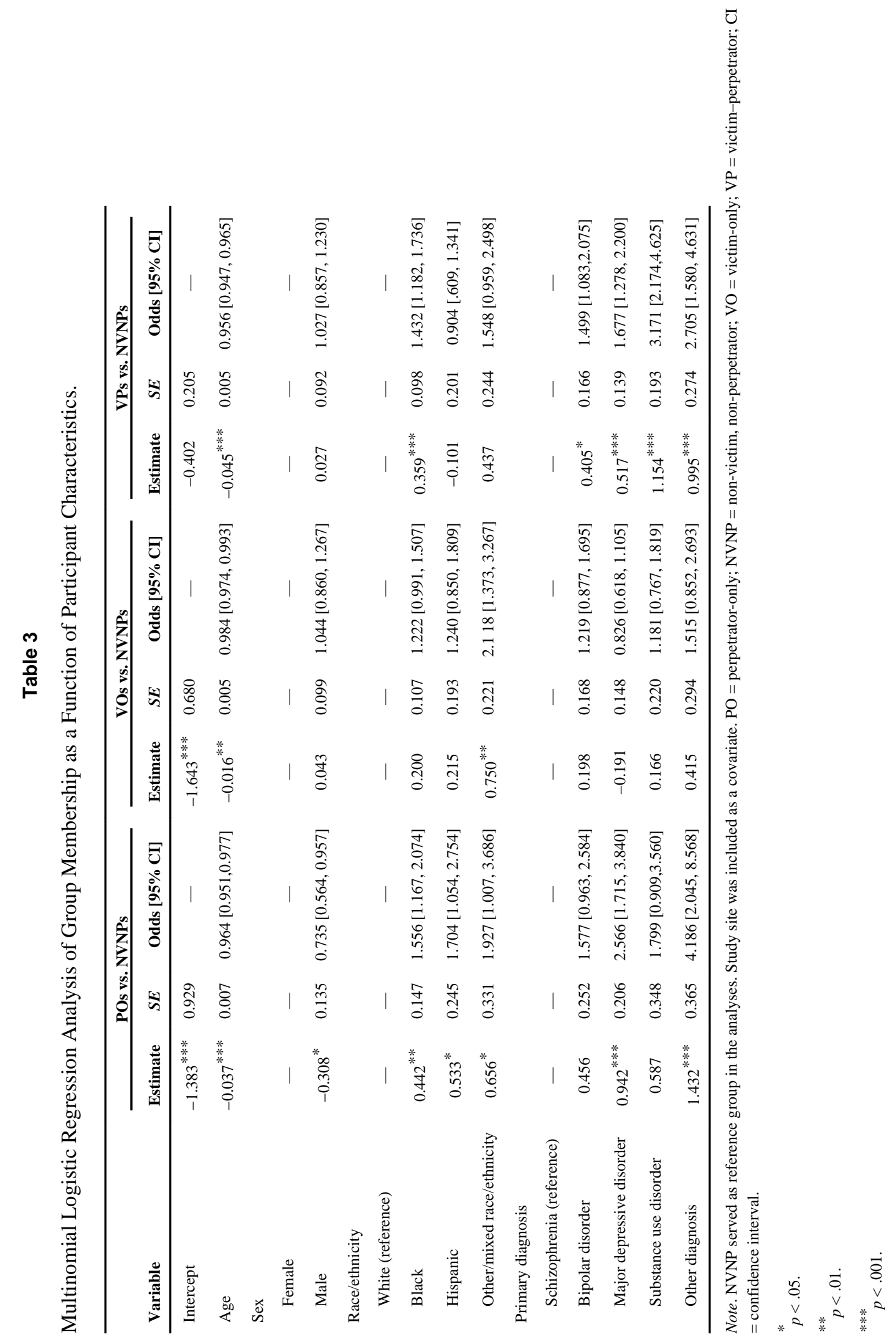

